\title{
Vector meson production at low $x$ from gauge/gravity duality
}

\author{
Miguel S. Costa, ${ }^{a}$ Marko Djurić ${ }^{a}$ and Nick Evans ${ }^{b}$ \\ ${ }^{a}$ Centro de Física do Porto, Departamento de Física e Astronomia, \\ Faculdade de Ciências da Universidade do Porto, \\ Rua do Campo Alegre 687, 4169-007 Porto, Portugal \\ ${ }^{b}$ STAG Research Centre, Physics and Astronomy, University of Southampton, \\ Highfield, Southampton, SO17,1BJ, U.K. \\ E-mail: miguelc@fc.up.pt, djuric@fc.up.pt, evans@soton.ac.uk
}

ABstract: We use gauge/gravity duality to study vector meson $\left(J / \Psi, \rho_{0}, \Omega, \Phi\right)$ production in electron-proton scattering, in the limit of high center of mass energy at fixed momentum transfer, corresponding to the limit of low Bjorken $x$, where the process is dominated by pomeron exchange. Our approach considers the pomeron at strong coupling, described by the graviton Regge trajectory in AdS space with a hard-wall to mimic confinement effects. Both the proton and vector mesons are described by simple holographic wave functions in AdS. This model agrees with HERA H1 data with a $\chi^{2}$ per degree of freedom below one on total cross-sections, and below two on differential cross-sections, confirming the success of previous studies that model low $x$ DIS and DVCS using gauge/gravity duality.

KEYwORDS: Gauge-gravity correspondence, AdS-CFT Correspondence, Phenomenological Models, QCD

ARXIV EPRINT: 1307.0009 


\section{Contents}

1 Introduction 1

2 Cross section for vector meson production 4

2.1 External kinematics 4

2.2 External state AdS wave functions 5

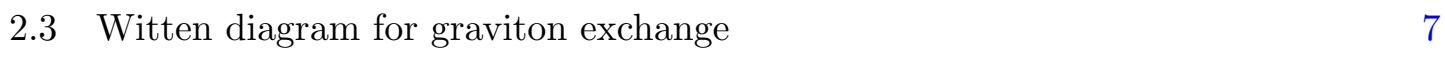

2.4 Exchange of graviton-Regge trajectory 8

$\begin{array}{lll}3 & \text { Results } & 10\end{array}$

\section{Introduction}

Vector meson production (VMP) is one of the diffractive processes studied in electronproton collisions at HERA. It is conceptually similar to deeply virtual Compton scattering (DVCS), but instead of an outgoing photon a vector meson is produced. The vector mesons have the same $J^{P C}$ values as the photon (i.e. $1^{--}$), so the process is kinematically similar. The key difference comes from the vector mesons' structure functions. Here we will study processes where the final state is a $\rho, \phi, J / \psi$ or $\Omega$. Figure 1 shows the process considered in this paper.

In the limit of high center of mass energy at fixed momentum transfer, corresponding to the limit of low Bjorken $x$, VMP is dominated by the exchange of the pomeron Regge

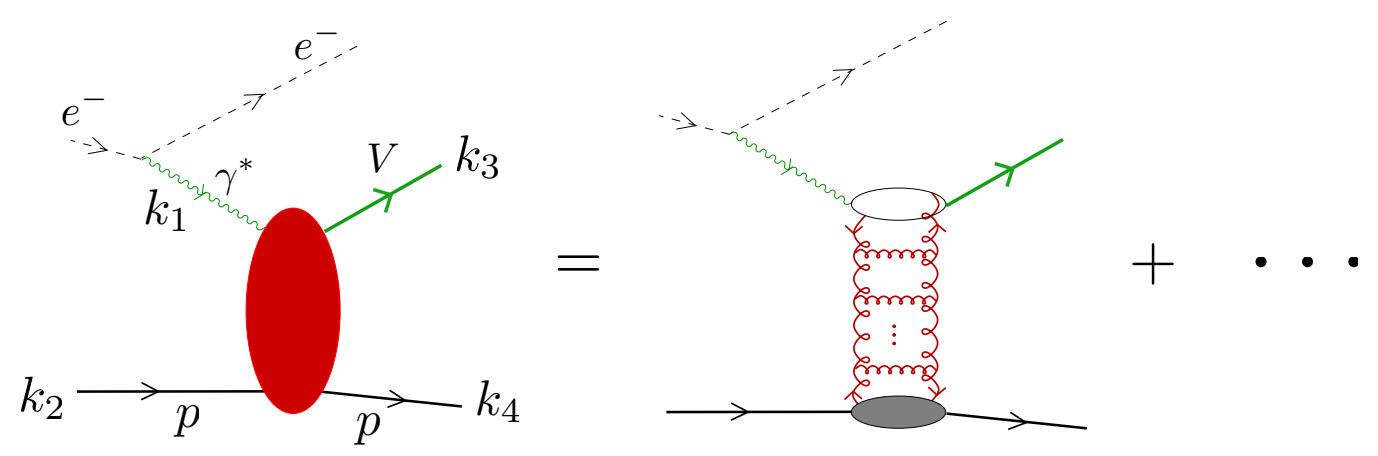

Figure 1. The process of vector meson production analysed in this paper. An off-shell photon of momenta $k_{1}$ (with $k_{1}^{2}=Q^{2}$ ) interacts with a proton $p$ of momenta $k_{2}$, leading to a final state of a scattered proton and a vector meson $V$. At low $x$ this process is dominated by the exchange of the pomeron, which at weak coupling can be described by the BFKL hard pomeron. 
trajectory between the photon and the proton. In particular, at large virtuality $Q^{2}$ of the incoming photon, diagrams of order $\left[\alpha_{s} \ln (1 / x)\right]^{n}$ can be resummed in perturbation theory and the process is described by the exchange of the hard pomeron [1-3], as shown schematically in figure 1. A number of authors [4-10] have studied vector meson production using the weak coupling analysis providing a decent fit to the data. More recently, the analysis in [11] uses AdS wave functions within a dipole approximation to fit $\rho$ production.

The Gauge/Gravity duality establishes a correspondence between the pomeron Regge trajectory and the graviton Regge trajectory of the dual string theory [12]. This correspondence has been used to study low- $x$ QCD processes dominated by pomeron exchange such as DIS [13-26] and DVCS [27-29]. In particular, it provides a description for the strong coupling expansion of pomeron exchange, whose intercept varies from $j_{0}=1$ at weak coupling to $j_{0}=2$ at strong coupling. Experimental evidence shows that, as one increases the coupling (by varying $Q^{2}$ from large to small), the effective spin of the exchanged pomeron grows from $j_{0} \sim 1.1$ to $j_{0} \sim 1.4$ (see for instance the figure presented in the conclusion of [25]). It is therefore conceivable that one may fit the data starting from the strong coupling regime, instead of the more conventional weak coupling BFKL approach. This is done here by considering the graviton Regge trajectory in AdS space. Confinement can be simply and successfully modelled by the inclusion of an infra-red hard-wall in this AdS space. A detailed discussion on the validity and assumptions behind this model was presented in [29], here we will be mainly concerned in computing the cross section for VMP at low $x$ using the dual AdS tree level diagram with the exchange of the graviton Regge trajectory, as shown in figure 2. We then compare to HERA data.

A new key aspect of our gauge/gravity duality description of VMP, in comparison with DIS and DVCS, will be to use a very simple holographic model for the vector mesons which gives the holographic wave function of the mesons as a function of their mass. These wave functions are normalisable modes of the AdS U(1) gauge field dual to the electromagnetic current operator $j_{f}^{a}=\bar{\psi}_{f} \gamma^{a} \psi_{f}$. Holographic models of vector mesons include using the D3/D7 system [30-34] and the Sakai-Sugimoto model [35, 36] - in each case a bulk gauge field is associated with the vector meson states and eigenvalues of its radial wave equation are computed with the AdS radius terminating at the scale of the constituent quark's mass. AdS/QCD is a phenomenological crystallization of these ideas [37, 38]. In coordinates where the AdS metric is given by $d s^{2}=\left(R^{2} / z^{2}\right)\left(d x^{2}+d z^{2}\right)$, for each meson we will simply solve for the radial wave function of an AdS vector field cut off at some radius $z_{f}$, which is determined by the physical mass of the meson. For most of the analysed data the overlap between the bulk-to-boundary propagator, which creates a source of the operator $j_{f}^{a}$ that couples to the virtual photon, the meson wave functions and the pomeron bulk-to-bulk propagator is in the UV region. In this region both the bulk-to-boundary propagator and the meson wave functions are determined by the canonical dimension of $j_{f}^{a}$. When the overlap moves to the QCD scale we rely on the simplest phenomenological model with a cutoff $z_{f}$ for the field dual to $j_{f}^{a}$, and also with an AdS hard-wall at a cut-off $z_{0}$ for the remaining fields. For the proton we assume that its wave function is highly peaked at a scale $z_{*}$.

The methodology we use here for pomeron exchange has already been developed and tested against experimental data in previous publications [17, 25, 29]. Holographic descrip- 


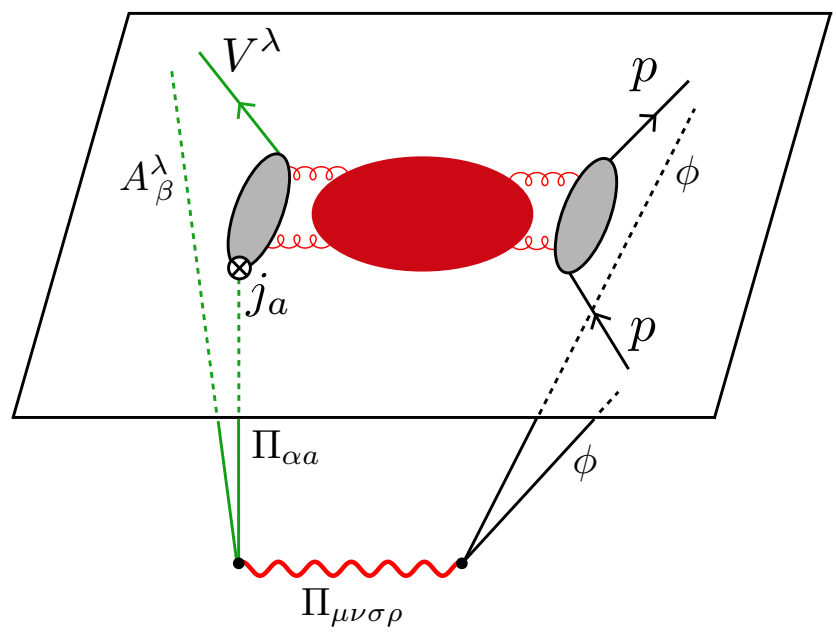

Figure 2. Holographic representation of the hadronic tensor needed to compute the cross section for VMP. Single pomeron exchange at strong coupling is described by the exchange of the AdS graviton Regge trajectory. In the light-cone coordinates used in this paper the exchange is dominated by the $\Pi_{++--}$component of the Reggeon propagator.

tions of vector mesons are discussed in detail in [34]. Hence, to keep this note short, we will quickly summarize the main results and new features needed for VMP, and then proceed to present the results. The reader is directed to the aforementioned sources for more information on using the AdS/CFT correspondence in the study of diffractive scattering.

It is however important to enumerate clearly the free parameters in our model. The meson wave functions are determined in terms of the scale $z_{f}$, but we fix this by the observed meson mass. Our wave function for the proton state includes the scale $z_{*}$ which we keep as a fit parameter. The Regge-graviton propagator is constructed from the conformal limit at strong coupling, where it depends on one parameter - the intercept $j_{0}$. There is one additional parameter that enters, $g_{0}^{2}$, which is determined by the coupling of the pomeron to the external states (and is therefore different for each vector final state). A fit to the data assuming the conformal propagator hence depends on three parameters and we find already a good fit. A fourth parameter can be introduced to represent confinement in the propagator, a hard-wall cut off at large $z_{0}>z_{f}$. We also make fits including this adjustment and find a better overall $\chi^{2}$ fit.

In the results section of the paper we present numerical fits of our model to HERA H1 data for $\rho, \phi$ and $J / \psi$ production as well as to the very limited ZEUS data for the $\Omega$. Our results are summarized in table 1 of section 3 . The overall fit to the total cross-sections has a $\chi^{2} /$ degree of freedom below one in each case, which confirms the approach captures the physics of the processes. The fits to the differential cross-sections have a $\chi^{2} /$ degree of freedom between one and two, which whilst not as good a fit as previously found for the DIS and DVCS using this approach, is still decent. 


\section{Cross section for vector meson production}

To compute the total cross section for vector meson production we need first to compute the following hadronic tensor

$$
W_{a}^{\lambda}\left(k_{j}\right)=i \int d^{4} y e^{i k_{1} \cdot y}\left\langle k_{3}, \lambda ; k_{4}\left|j_{a}(y)\right| k_{2}\right\rangle,
$$

where $\lambda$ is the polarization of the outgoing vector meson and we refer to figure 1 for the kinematics. Contracting with the polarization of the incoming photon, the amplitude for the transition between a photon of polarization $\lambda$ and a vector meson of polarization $\lambda^{\prime}$ is

$$
W^{\lambda \lambda^{\prime}}\left(k_{j}\right)=\left(n^{\lambda}\right)^{a} W_{a}^{\lambda^{\prime}}\left(k_{j}\right) .
$$

We will average over the incoming polarizations and sum over the final ones. The differential cross-section is then given by

$$
\frac{d \sigma}{d t}\left(x, Q^{2}, t\right)=\frac{1}{16 \pi s^{2}} \frac{1}{3} \sum_{\lambda, \lambda^{\prime}=1}^{3}\left|W^{\lambda \lambda^{\prime}}\right|^{2} .
$$

We will compute $W^{\lambda \lambda^{\prime}}$ from the AdS Witten diagram sketched in figure 2. We will now discuss the key elements of such diagram.

\subsection{External kinematics}

Let us describe the kinematics of the external particles. We use light-cone coordinates $(+,-, \perp)$, with metric given by $d s^{2}=-d x^{+} d x^{-}+d x_{\perp}^{2}$, where $x_{\perp} \in \mathbb{R}^{2}$ is a vector in impact parameter space. For the incoming particles we take

$$
k_{1}=\left(\sqrt{s},-\frac{Q^{2}}{\sqrt{s}}, 0\right), \quad k_{2}=\left(\frac{M^{2}}{\sqrt{s}}, \sqrt{s}, 0\right),
$$

where $M$ is the mass of the target and the incoming off-shell photon is space-like with $k_{1}^{2}=Q^{2}>0$. For the outgoing particles

$$
k_{3}=-\left(\sqrt{s}, \frac{q_{\perp}^{2}+m^{2}}{\sqrt{s}}, q_{\perp}\right), \quad k_{4}=-\left(\frac{M^{2}+q_{\perp}^{2}}{\sqrt{s}}, \sqrt{s},-q_{\perp}\right)
$$

where $m$ is the mass of the vector meson that is created. We consider the Regge limit of large $s$ and fixed $t=-q_{\perp}^{2}$.

We also need to define the photon and vector meson polarization vectors. Let $n_{\lambda}$ and $n_{\lambda}^{\prime}$ be, respectively, the photon and vector meson polarization vectors. We can use the gauge freedom to impose the conditions

$$
n \cdot k_{1}=0, \quad n^{\prime} \cdot k_{3}=0 .
$$

We normalize the photon polarization such that $n^{2}=1$ for transverse polarizations and $n^{2}=-1$ for longitudinal polarization (the photon is space-like). For the vector meson we have always $n^{\prime 2}=1$. 
In the above light-cone coordinates, the polarization of the transverse incoming photon is

$$
n_{\lambda}=\left(0,0, \epsilon_{\lambda}\right), \quad(\lambda=1,2)
$$

where $\epsilon_{\lambda}$ is an orthogonal basis of unit vectors on $\mathbb{R}^{2}$. The incoming longitudinal photon has polarization

$$
n_{3}=\frac{1}{Q}\left(\sqrt{s}, \frac{Q^{2}}{\sqrt{s}}, 0\right)
$$

where we define $Q=\sqrt{Q^{2}}$. Note that all the $n_{i}$ are orthogonal (and unit normalized).

For the outgoing meson we introduce the two (transverse) polarizations

$$
n_{\lambda}^{\prime}=\left(0,2 \frac{\epsilon_{\lambda}^{\prime} \cdot q_{\perp}}{\sqrt{s}}, \epsilon_{\lambda}^{\prime}\right), \quad(\lambda=1,2)
$$

where $\epsilon_{\lambda}^{\prime}$ is an orthogonal basis of unit vectors on $\mathbb{R}^{2}$. This polarization is transverse, in the sense that in the Regge limit the leading component is on the transverse space $\mathbb{R}^{2}$. For the other polarization we take

$$
n_{3}^{\prime}=\frac{1}{m}\left(\sqrt{s}, \frac{-m^{2}+q_{\perp}^{2}}{\sqrt{s}}, q_{\perp}\right)
$$

This polarization is longitudinal, in the sense that in the Regge limit the leading component is light-like and parallel to the meson momenta. Again all $n_{i}^{\prime}$ are orthogonal (and unit normalized).

\subsection{External state AdS wave functions}

In the gravity dual description bound states are associated with eigenmode wave functions in the holographic $z$ direction. The vector mesons for example are described by a normalizable mode of the AdS U(1) gauge field dual to the electromagnetic current operator $j_{f}^{a}=\bar{\psi}_{f} \gamma^{a} \psi_{f}$. The non-normalizable mode is dual to this operator's source (which couples to the virtual photon when we include the QED coupling to the quarks). To fix the asymptotic normalization of the mode we must impose a large $z$ (IR) boundary condition on the solution. We simply include a "fermion hard-wall" at the scale $z_{f} \sim m_{f}^{-1}$ for each fermion flavour and impose Neumann boundary conditions on the field at the wall. The value of $z_{f}$ is phenomenologically fixed by the measured vector meson mass and the model contains no free parameters in the mesonic sector.

We write the normalizable mode for the AdS gauge field describing the vector mesons as

$$
A_{\mu}^{\lambda}(X)=\int \frac{d^{4} k}{(2 \pi)^{4}} n_{\mu}^{\prime \lambda} e^{i k \cdot x} A(z, k)
$$

where $X=(z, x)$ are the usual Poincaré coordinates in AdS. In the gauge $D_{\mu} A^{\mu}=0$, the field equation $D^{2} A_{\mu}=0$ is solved by

$$
A(z, k)=\frac{\sqrt{2}}{\xi J_{1}(\xi)} m z J_{1}(m z), \quad k^{2}=-m^{2},
$$


with the polarisation vector satisfying

$$
n_{z}^{\prime}=0, \quad n_{a}^{\prime} k^{a}=0
$$

where the boundary polarisation $n_{a}^{\prime}$ is given by either (2.9) or (2.10). We have taken the normalizable Bessel function solution, so that the wave function falls off asymptotically as $z^{2}$, as required for a massless vector field dual to the vector operator $j_{f}^{a}$ of dimension 3. The constant $\xi=2.4048 \ldots$ in the expression (2.12) for $A(z, k)$ is the first zero of the Bessel funcion $J_{0}$ and arises from imposing Neumann boundary conditions on the field at the cut-off scale $z_{f}$ defined by the quark cut off scale $z_{f} \sim 1 / m_{q_{f}}$. The relation between the meson mass and the corresponding quark cut off is then $m=\xi / z_{f}$. The overall constant in $A(z, k)$ follows from the normalization condition

$$
\int_{0}^{z_{f}} \frac{d z}{z}|A(z, k)|^{2}=1
$$

To compute the hadronic tensor (2.1) we need to compute the expectation value of the operator $j_{f}^{a}$ in the state defined by the incoming proton, and outgoing proton and vector meson. Thus we need to include the bulk-to-boundary propagator of the same gauge field, given by its non-normalizable mode, which is dual to a source of $j_{f}^{a}$ (see figure 2) and behaves asymptotically as $z^{0}$. Denoting the propagator between the bulk point $X=(z, x)$ and the boundary point $y$ by $\Pi_{\mu a}(X, y)$, in the gauge $D_{\mu} A^{\mu}=0$ we have

$$
\Pi_{z a}(X, y)=0, \quad \Pi_{b a}(X, y)=\int \frac{d^{4} k}{(2 \pi)^{4}} e^{i k \cdot(x-y)} \Pi_{b a}(z, k),
$$

with

$$
\Pi_{b a}(z, k)=\sqrt{C \frac{\pi^{2}}{6}}\left(\eta_{b a}-\frac{k_{b} k_{a}}{k^{2}}\right) Q z K_{1}(Q z), \quad k^{2}=-Q^{2} .
$$

Note that this is the solution in a conformal theory that extends to infinite $z$. Using the same simple model as above, we should also introduce Neumann boundary conditions at the IR wall located at $z=z_{f}$, as described in [39]. However, a simple analysis of the overlap between the vector meson wave function and the bulk-to-boundary propagator shows that this modification is only important when the vector meson mass $m$ is of the same order as the off-shellness $Q$. This does not happen for most of the data points here considered. Indeed we have found changes in the IR boundary conditions of the bulk-to-boundary propagator to have little impact on the fits.

The constant $C$ in (2.16) is fixed by the normalization of the current operator twopoint function given by $\left\langle j_{a}(y) j_{b}(0)\right\rangle=\left(C / y^{6}\right)\left(\eta_{a b}-2 y_{a} y_{b} / y^{2}\right)$. In free theory, for a quark of charge $q_{f}$, we have $C=3 \alpha q_{f}^{2} / \pi^{3}$, where $\alpha=e^{2} /(4 \pi)$ is the fine structure constant. To fix a reference value for this normalization constant we assume the flavour content of our vector mesons is given by $\rho=\frac{1}{\sqrt{2}}(u \bar{u}-d \bar{d}), \Omega=\frac{1}{\sqrt{2}}(u \bar{u}+d \bar{d}), \Phi=s \bar{s}$ and $J / \psi=c \bar{c}$. We then have $C_{\rho}=C_{\Omega}=5 \alpha /\left(6 \pi^{3}\right), C_{\Phi}=\alpha /\left(3 \pi^{3}\right)$ and $C_{J / \psi}=4 \alpha /\left(3 \pi^{3}\right)$. Since over the kinematical range of VMP here considered the theory is not free, our fits will also allow for changes from this reference values. 
At the opposite vertex of the t-channel exchange a proton scatters and in principle one should put the AdS wave functions for both incoming and outgoing protons. For example, we could describe the proton by the normalizable mode of a scalar in AdS with mass squared of $9 / 4$. The solution, $\phi(z) \propto z^{3 / 2}(-\sin z-3 \cos z / z+3 \sin z / z)$, would fall off as $z^{9 / 2}$ matching the dimension of $\psi_{f}^{3}$. This behaviour would extend up to some scale $z_{*}$ associated with the dynamical mass of the proton's constituents. In fact, since the proton wave function is sufficiently fast falling at small $z$, we will take simply a delta function localized at a scale $z_{*}$. More concretely we consider

$$
\Phi(z)=|\phi(z)|^{2}=z^{3} \delta\left(z-z_{*}\right),
$$

which satisfies the normalization condition

$$
\int \frac{d z}{z^{3}}|\phi(z)|^{2}=1
$$

We will leave $z_{*}$ as a free parameter in our fits. An additional benefit of this approach is that the $z$ integration at the vertex can be done analytically rather than numerically, greatly reducing the numerical computation time for the process. This is a considerable saving across many fits. In fact we did try some sample runs using the full proton wave function but found only a very small change in the fit parameters.

\subsection{Witten diagram for graviton exchange}

In the Regge limit, the amplitude for $\gamma^{*} p \rightarrow V p$, computed from the dual Witten diagram in AdS, is dominated by t-channel exchange of the graviton Regge trajectory. Let us first consider the limit of very large 't Hooft coupling, where graviton exchanges dominates the other massive strings in this trajectory. In this case, for the above external Regge kinematics, standard Feynman rules in AdS give [40-44]

$$
\begin{aligned}
W_{a}^{\lambda}\left(k_{i}\right)= & \kappa^{2} \int d l_{\perp} e^{i q_{\perp} \cdot l_{\perp}} \int \frac{d z}{z^{3}} \frac{d \bar{z}}{\bar{z}^{3}} 2 \partial_{1[-} \Pi_{\alpha] a}\left(z, k_{1}\right) g^{\alpha \beta}(z) 2 \partial_{3[-} A_{\beta]}^{\lambda}\left(z, k_{3}\right) \\
& i k_{2+} \phi\left(\bar{z}, k_{2}\right) i k_{4+} \phi\left(\bar{z}, k_{4}\right) 16 z \bar{z} \Pi_{\perp}(L)
\end{aligned}
$$

where we used the notation

$$
2 \partial_{1[-} \Pi_{\alpha] a}\left(z, k_{1}\right)=\sqrt{C \frac{\pi^{2}}{6}} \times\left\{\begin{array}{ll}
2 i k_{1[-} \eta_{c] a} Q z K_{1}(Q z), & \alpha=c \\
\left(\eta_{-a}-\frac{k_{1-} k_{1 a}}{Q^{2}}\right) Q^{2} z K_{0}(Q z), & \alpha=z
\end{array},\right.
$$

and

$$
2 \partial_{3[-} A_{\beta]}^{\lambda}\left(z, k_{3}\right)=\frac{\sqrt{2}}{\xi J_{1}(\xi)} \times\left\{\begin{array}{ll}
2 i k_{3[-} n_{d]}^{\prime \lambda} m z J_{1}(m z), & \beta=d \\
-n_{-}^{\prime \lambda} m^{2} z J_{0}(m z), & \beta=z
\end{array} .\right.
$$

We have discussed in the previous section the form of the bulk-to-boundary propagator of the AdS vector field and also of the normalizable modes dual to the vector meson and to the proton.

The above amplitude already takes into account that for the Regge kinematics here considered the component $\Pi_{++--}$of the graviton propagator dominates the exchange, and 
can be integrated along the light cone directions to give a scalar propagator $\Pi_{\perp}(L)$ on the AdS transverse space $H_{3}$ of mass squared 3 [40], with

$$
\cosh L=\frac{z^{2}+\bar{z}^{2}+l_{\perp}^{2}}{2 z \bar{z}} .
$$

Next we contract the amplitude $W_{a}^{\lambda^{\prime}}\left(k_{i}\right)$ with the incoming photon polarization $n^{\lambda}$, and then consider all possible polarizations $\lambda$ and $\lambda^{\prime}$. It turns out that the non-zero contributions preserve helicity, i.e. $W_{L T}=0=W_{T L}$. A simple computation shows that

$$
\begin{aligned}
& W_{T T}=\left(n^{\lambda}\right)^{a} W_{a}^{\lambda^{\prime}}\left(k_{j}\right)=\left(\epsilon_{\lambda} \cdot \epsilon_{\lambda^{\prime}}\right) Q m W_{1}, \quad\left(\lambda, \lambda^{\prime}=1,2\right) \\
& W_{L L}=\left(n^{\lambda}\right)^{a} W_{a}^{\lambda^{\prime}}\left(k_{j}\right)=-Q m W_{0}, \quad\left(\lambda=\lambda^{\prime}=3\right)
\end{aligned}
$$

where

$$
W_{n}=2 i s \int d l_{\perp} e^{i q_{\perp} \cdot l_{\perp}} \int \frac{d z}{z^{3}} \frac{d \bar{z}}{\bar{z}^{3}} \Psi_{n}(z) \Phi(\bar{z})\left[i \frac{\kappa^{2}}{2} S \Pi_{\perp}(L)\right],
$$

with $S=z \bar{z} s$,

$$
\Psi_{n}(z)=-\left(\sqrt{\frac{C \pi^{2}}{6}} z^{2} K_{n}(Q z)\right)\left(\frac{\sqrt{2}}{\xi J_{1}(\xi)} z^{2} J_{n}(m z)\right),
$$

and $\Phi(\bar{z})$ is given in $(2.17)$ above.

\subsection{Exchange of graviton-Regge trajectory}

So far we have evaluated the Witten diagram with a single graviton exchange which is valid for very large 't Hooft coupling $\lambda$. To extend this result to smaller coupling/string tension, including the correction from string states in the leading Regge trajectory, we first need to realise that the amplitude (2.25) can be written in terms of a conformal amplitude $\mathcal{B}(S, L)$. Taking care of the external polarizations, one can then show that the amplitude $W_{n}$ defined in (2.25) becomes

$$
W_{n}=2 i s \int d l_{\perp} e^{i q_{\perp} \cdot l_{\perp}} \int \frac{d z}{z^{3}} \frac{d \bar{z}}{\bar{z}^{3}} \Psi_{n}(z) \Phi(\bar{z}) \mathcal{B}(S, L) .
$$

This general form relies only on conformal invariance, as shown in [44] and reviewed at length in [29]. At very large 't Hooft coupling, introducing the AdS phase shift $\chi(S, L)=$ $i \mathcal{B}(S, L)$, the Witten diagram for single graviton exchange in the Regge limit has

$$
\chi(S, L)=-\frac{\kappa^{2}}{2} S \Pi_{\perp}(L) .
$$

In this paper we are interested in the limit of large 't Hooft coupling $\lambda \gg 1$, but with sufficiently high energies such that $\sqrt{\lambda} / \ln S \ll 1$. In this limit all fields in the graviton Regge trajectory contribute to the amplitude [45], and we have

$$
\mathcal{B}(S, L)=g_{0}^{2}\left(1+i \cot \left(\frac{\pi \rho}{2}\right)\right)\left(\alpha^{\prime} S\right)^{1-\rho} \frac{e^{-\frac{L^{2}}{\rho \ln \left(\alpha^{\prime} S\right)}}}{\left(\rho \ln \left(\alpha^{\prime} S\right)\right)^{3 / 2}} \frac{L}{\sinh L}
$$


where

$$
\alpha^{\prime} S=\frac{z \bar{z} s}{\sqrt{\lambda}}, \quad \rho=2-j_{0}=\frac{2}{\sqrt{\lambda}} .
$$

In this equation the 't Hooft coupling is defined from the AdS radius as $\lambda=R^{4} / \alpha^{\prime 2}$, where $\alpha^{\prime}$ is the tension of the dual QCD string ${ }^{1}$. The coupling $g_{0}^{2}$ is related to the impact factors of the external states. The resulting graviton-Regge amplitude depends on two parameters: the intercept $j_{0}$ (or equivalently the above defined 't Hooft coupling $\lambda$ ) and the coupling $g_{0}^{2}$. Note that, as an overall coefficient of the Feynman diagram, $g_{0}^{2}$ also soaks up any freedom in the normalization constant $C$ of the $\mathrm{U}(1)$ gauge field bulk-to-boundary propagator. In other words, the fits to data will fix only the combination $\sqrt{C} g_{0}^{2}$, and for this reason we shall fix, when presenting the results, $C$ to the reference values given in section 2.2.

As so far presented the graviton-Regge, or the BPST pomeron [12], trajectory exchanged in AdS is that of a conformal theory. In QCD we expect that confinement will enter in the IR and cut off any deep IR contribution to the process. The simplest model of that effect is just to include a hard-wall in $z$ at $z_{0}$ on the Pomeron propagator. We will introduce this extra parameter into a second fit to the data below also. In this model, the propagator for the Pomeron is modified, due to the different boundary conditions in the differential equation that defines it [12]. In our analysis, following [25], we use the approximation (with $\tau=\log \left(\alpha^{\prime} S\right)$ )

$$
\chi_{h w}\left(\tau, l_{\perp}, z, \bar{z}\right)=C(\tau, z, \bar{z}) D\left(\tau, l_{\perp}\right) \chi_{h w}^{(0)}\left(\tau, l_{\perp}, z, \bar{z}\right),
$$

where

$$
D\left(\tau, l_{\perp}\right)=\min \left(1, \frac{\exp \left[-m_{1} l_{\perp}-\left(m_{0}-m_{1}\right)^{2} l_{\perp}^{2} / 4 \rho \tau\right]}{\exp \left[-m_{1} z_{0}-\left(m_{0}-m_{1}\right)^{2} z_{0}^{2} / 4 \rho \tau\right]}\right),
$$

is an exponential cutoff at large $l_{\perp}$, known to be present asymptotically and determined by the first glueball masses $m_{0}$ and $m_{1}$, and

$$
\chi_{h w}^{(0)}\left(\tau, l_{\perp}, z, \bar{z}\right)=\chi_{c}\left(\tau, l_{\perp}, z, \bar{z}\right)+\mathcal{F}(\tau, z, \bar{z}) \chi_{c}\left(\tau, l_{\perp}, z, z_{0}^{2} / \bar{z}\right) .
$$

In the above $\chi_{c}\left(\tau, l_{\perp}, z, \bar{z}\right)$ is derived from the conformal kernel, equation (2.29). This approximation stems from the fact that the hard-wall kernel can be shown to have the above form at $t=q_{\perp}^{2}=0$, i.e. with $l_{\perp}$ integrated over while setting $q_{\perp}=0$, and then extrapolating to $l_{\perp}$. $C\left(\tau, z, z^{\prime}\right)$, is a normalization function, independent of $l_{\perp}$, which ensures the $t=0$ result is retained. For details see [25, 29]. The function

$$
\mathcal{F}(\tau, z, \bar{z})=1-4 \sqrt{\pi \tau} e^{\eta^{2}} \operatorname{erfc}(\eta), \quad \eta=\frac{-\log \left(z \bar{z} / z_{0}^{2}\right)+4 \tau}{\sqrt{4 \tau}},
$$

is set by the boundary conditions at the wall and represents the relative importance of the two terms and therefore confinement. This function varies between -1 and 1 , approaching -1 at either large $z$, which roughly corresponds to small $Q^{2}$, or at large $\tau$ corresponding to small $x$. It is therefore in these regions that confinement is important.

\footnotetext{
${ }^{1}$ In $\mathcal{N}=4 \mathrm{SYM}, \lambda=g_{Y M}^{2} N$ is also the 't Hooft coupling defined from perturbation theory. On the other hand, in the kinematic range analysed in this paper, where we start by considering that in this range QCD is approximately conformal, just as in the weak coupling BFKL analysis, the precise relation between $\lambda=R^{4} / \alpha^{\prime 2}$ and the 't Hooft coupling defined from perturbation theory is not known. This would mean finding the dual QCD string.
} 


\begin{tabular}{|c|c|c|c|c|c|c|c|c|}
\hline & \multicolumn{4}{|c|}{$\sigma[\mathrm{nb}]$} & \multicolumn{3}{|c|}{$\mathrm{d} \sigma / \mathrm{dt}\left[\mathrm{nb} / \mathrm{GeV}^{2}\right]$} \\
\hline & & $\rho$ & $\phi$ & $\Omega$ & $\mathrm{J} / \psi$ & $\rho$ & $\phi$ & $\mathrm{J} / \psi$ \\
\hline & $m$ [GeV] & 0.77549 & 1.019445 & 0.78265 & 3.096916 & 0.77549 & 1.019445 & 3.096916 \\
\hline & $\mathrm{N}$ & 48 & 27 & 6 & 38 & 35 & 21 & 84 \\
\hline $\begin{array}{l}\mathrm{C} \\
\mathrm{O}\end{array}$ & $\alpha^{2}$ & 0.92 & 0.60 & 0.0099 & 0.28 & 1.7 & 1.3 & 2.9 \\
\hline $\begin{array}{l}n \\
f \\
o\end{array}$ & $g_{0}^{2}$ & 4.6 & 1.8 & 0.53 & 0.62 & 1.6 & 0.25 & 0.56 \\
\hline $\begin{array}{l}r \\
m\end{array}$ & $\rho$ & 0.76 & 0.73 & 0.64 & 0.70 & 0.65 & 0.54 & 0.72 \\
\hline $\begin{array}{l}a \\
1\end{array}$ & $z^{*}\left[\mathrm{GeV}^{-1}\right]$ & 3.4 & 3.0 & 1.8 & 0.98 & 2.1 & 2.5 & 2.2 \\
\hline $\mathrm{H}$ & $\alpha^{2}$ & 0.88 & 0.61 & 0.015 & 0.30 & 1.7 & 1.4 & 1.8 \\
\hline $\begin{array}{l}a \\
r\end{array}$ & $g_{0}^{2}$ & 4.1 & 1.8 & 0.67 & 0.75 & 2.2 & 0.38 & 0.69 \\
\hline $\begin{array}{l}d \\
w\end{array}$ & $\rho$ & 0.76 & 0.73 & 0.66 & 0.71 & 0.69 & 0.59 & 0.75 \\
\hline $\begin{array}{l}a \\
1\end{array}$ & $z^{*}\left[\mathrm{GeV}^{-1}\right]$ & 3.6 & 3.6 & 1.5 & 0.87 & 2.2 & 2.5 & 2.4 \\
\hline 1 & $z_{0}\left[\mathrm{GeV}^{-1}\right]$ & 4.8 & 4.4 & 7.3 & 5.3 & 7.7 & 8.6 & 4.6 \\
\hline
\end{tabular}

Table 1. Output data for our fits showing the number of experimental points, $N$, the $\chi^{2}$ per degree of freedom for the fit and the best fit parameter values for $j_{0}=2-\rho$ (the intercept), $g_{0}^{2}$ (a vertex factor fitted for each meson state, with respect to reference values of the normalisation constants $C$ given in section 2.2), $z^{*}$ (the IR scale characterizing the proton wave function) and, for the hard-wall model fits, $z_{0}$ (an IR confinement cut-off).

\section{Results}

Our tree level AdS Feynman diagram provides a full description of the low $x$ vector meson production in terms of the parameters $j_{0}=2-\rho$ (the intercept), $g_{0}^{2}$ (a vertex factor fitted for each meson state), $z^{*}$ (the IR scale characterizing the proton wave function) and if we choose $z_{0}$ (an IR confinement cut off). We will now perform a fit to the data collected at HERA by the H1 collaboration $[46,47]$.

All of the data is at small $x(<0.01)$. Note that the $\rho, \phi$ and $\Omega$ have a mass close to each other, ranging from $\sim 0.78-1.02 \mathrm{GeV}$, whereas the $J / \Psi$ has a significantly higher mass at $3.09 \mathrm{GeV}$. This means that it is precisely for the $J / \Psi$ that the flavour cut-off scale becomes closer to the probe scale $Q$, therefore indicating that details of the AdS model for this vector meson will be be more important.

In table 1 we see a summary of all our fits. We show fits to the full cross-section and the differential cross-sections for each process. $N$ labels the number of available data points. We list the $\chi^{2}$ per degree of freedom in the fit and the best fit values of the parameters. Although data is available from the ZEUS collaboration as well [48-51], we 

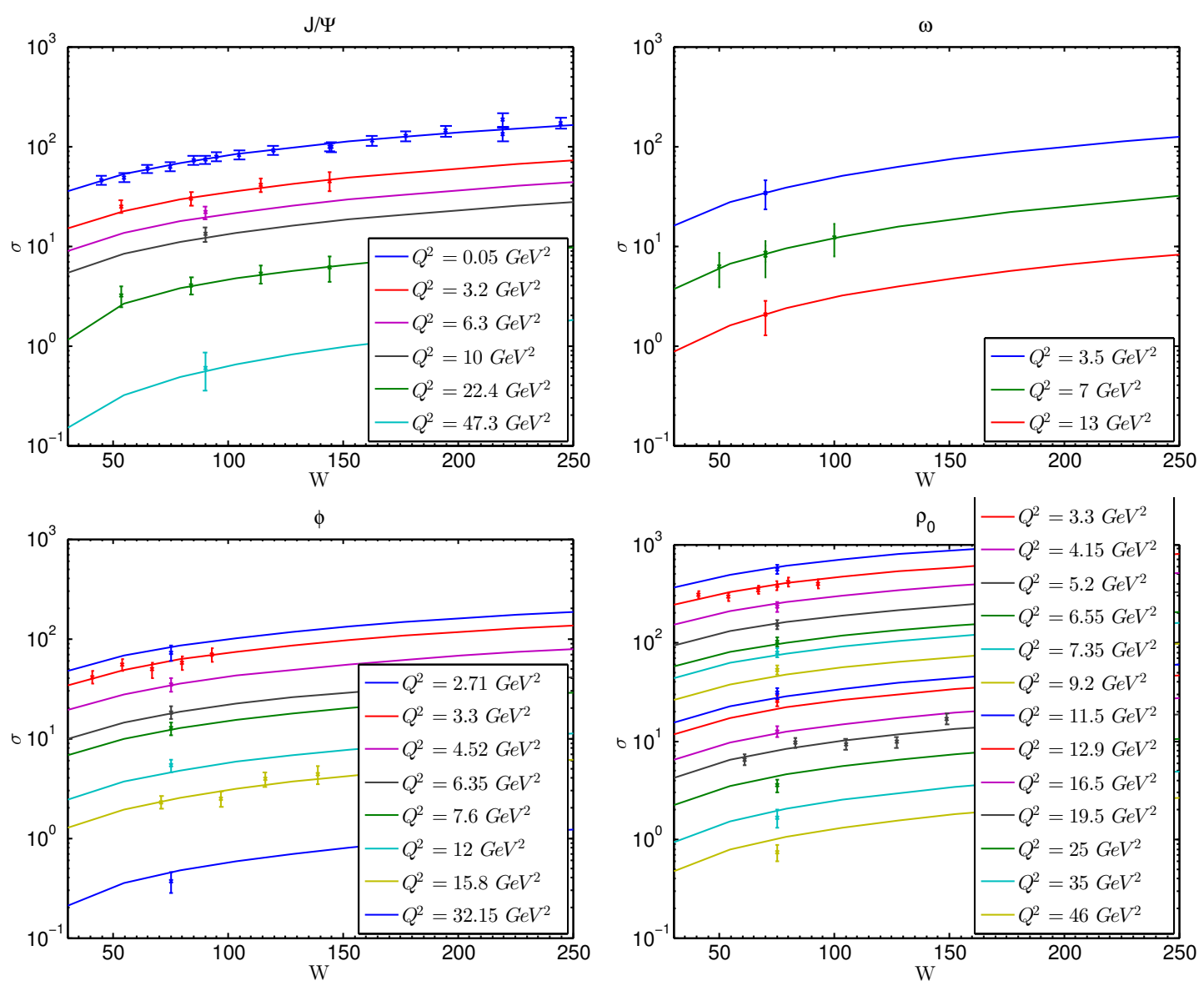

Figure 3. Cross sections using the conformal model.

find that in fitting the differential cross section we obtain better fits using the H1 data. This is most pronounced in the fit for the $J / \Psi$ meson where fitting the H1 differential cross section we get the results from table 1, while fitting just the ZEUS data we get a $\chi_{\text {d.o.f. }}^{2}=9.57$. Fitting both $\mathrm{H} 1$ and ZEUS together for this meson (but without taking into account the normalization differences between the data sets) we get a $\chi_{\text {d.o.f. }}^{2}=6.74$. For the remaining mesons fitting the data from both collaborations together gives only a slightly worse $\chi_{\text {d.o.f. }}^{2}$, but to be consistent throughout we chose to present the fits and plots for just the $\mathrm{H} 1$ data $^{3}$. If the measurements of both collaborations are properly statistically combined in the manner of [52], with corresponding fits given in [25] for the case of DIS, it would be possible to revisit the fits here presented. It is also interesting to note that the H1 data set for $J / \Psi$ goes to the lowest value of $Q^{2}$, hence giving us the most direct probe of our hard-wall model near the location of the cutoff.

Firstly, the fits to the full cross-sections provide very good $\chi^{2}<1$ in all cases. We

\footnotetext{
${ }^{2}$ For individual data sets, H1 gives a better fit in each case.

${ }^{3}$ The only exception is the cross section for the $\Omega$ meson, where we could only find ZEUS data.
} 

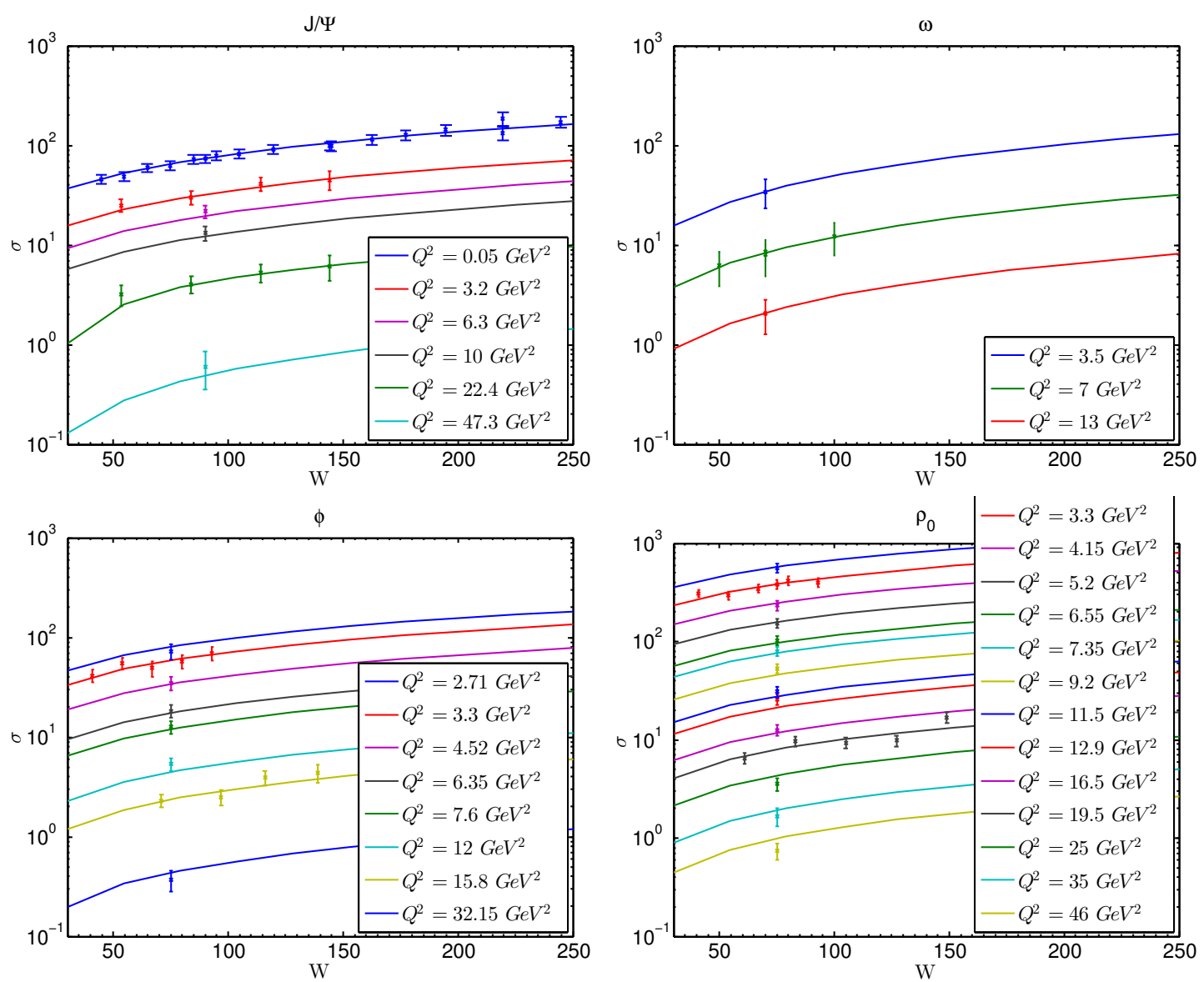

Figure 4. Cross sections using the hard-wall model.

display the fit to a representative sample of the data points ${ }^{4}$ in figures 3 and 4 . Note the $\Omega$ production fit is only to 6 data points. The best fit for the intercept $j_{0}=2-\rho$ is in the range $0.64<\rho<0.76$ across the fits, which seems fairly stable, and consistent with the intercepts found in DIS [25] and DVCS [29]. Note that, if we naively use the $\mathcal{N}=4$ SYM result $\rho=2 / \sqrt{g_{Y M}^{2} N}$, this corresponds to fit values for $\alpha_{s}$ between 0.18 and 0.26 . The best fit values for $g_{0}^{2}$ vary by almost an order of magnitude but since these are couplings specific to the meson states, that differ in each process, there is therefore no expectation that they should precisely match. The best fit values for the position of the hard-wall in the proton wave function, $z_{*}$, vary by a factor of 4 or so across the fits (giving a typical value of $500 \mathrm{MeV}$ for the wall position). The inclusion of the hard-wall cut-off parameter $z_{0}$ has very little impact on the goodness of fit in any of the cases (a fairly stable value around $200 \mathrm{MeV}$ emerges).

We can now turn to our fits of differential cross-section data (which is not available for the $\Omega$ meson). To avoid cluttering the paper with too many figures, we display the fit to a

\footnotetext{
${ }^{4}$ Of course, in table 1 all of the values correspond to the fit to all the points.
} 

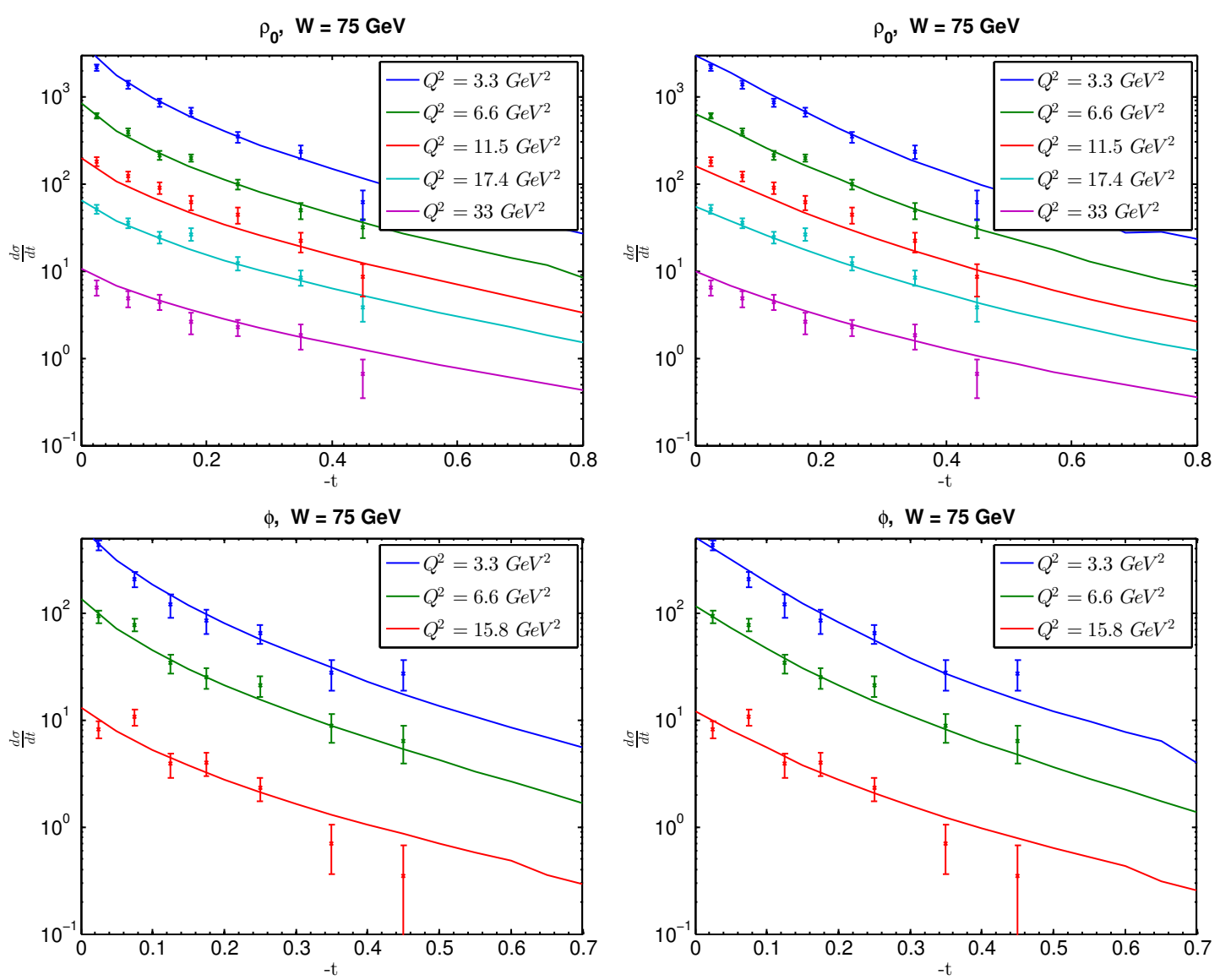

Figure 5. Differential cross section for the $\rho$ meson (top plots) and for the $\phi$ meson (bottom plots). Note that even though the plots for each meson appear very similar, the left hand ones use the conformal Pomeron, and the right hand ones use the hard-wall Pomeron.

representative sample of the data points in figures 5 and 6 . The fits are less good than for the full cross-section data but still have $\chi^{2}<2$ in each case. To claim such a good fit for the $J / \Psi$ meson we do need to include the hard-wall parameter $z_{0}$ and this is the only place in our fits where it makes a significant impact. For this process the momentum transfer energies $t$ go as low as $0.05 \mathrm{GeV}^{2}$, which is already below the hard-wall cut off scale set by $1 / z_{0}$. We therefore might need to improve the hard-wall model in order to obtain a better fit for this meson. We also note that these fits are not quite as good as the equivalent ones to DIS and DVCS data using the AdS methods, presumably reflecting the additional complication of fitting the mesonic wave functions holographically. The fit parameters show the same broad behaviour as for the full cross-section although $z^{*}$ seems more stable.

In conclusion we find that the strong coupling AdS/CFT inspired model of low $x$ vector meson production gives a very good fit to the data, providing further evidence for the strength of gauge gravity duality methods. 

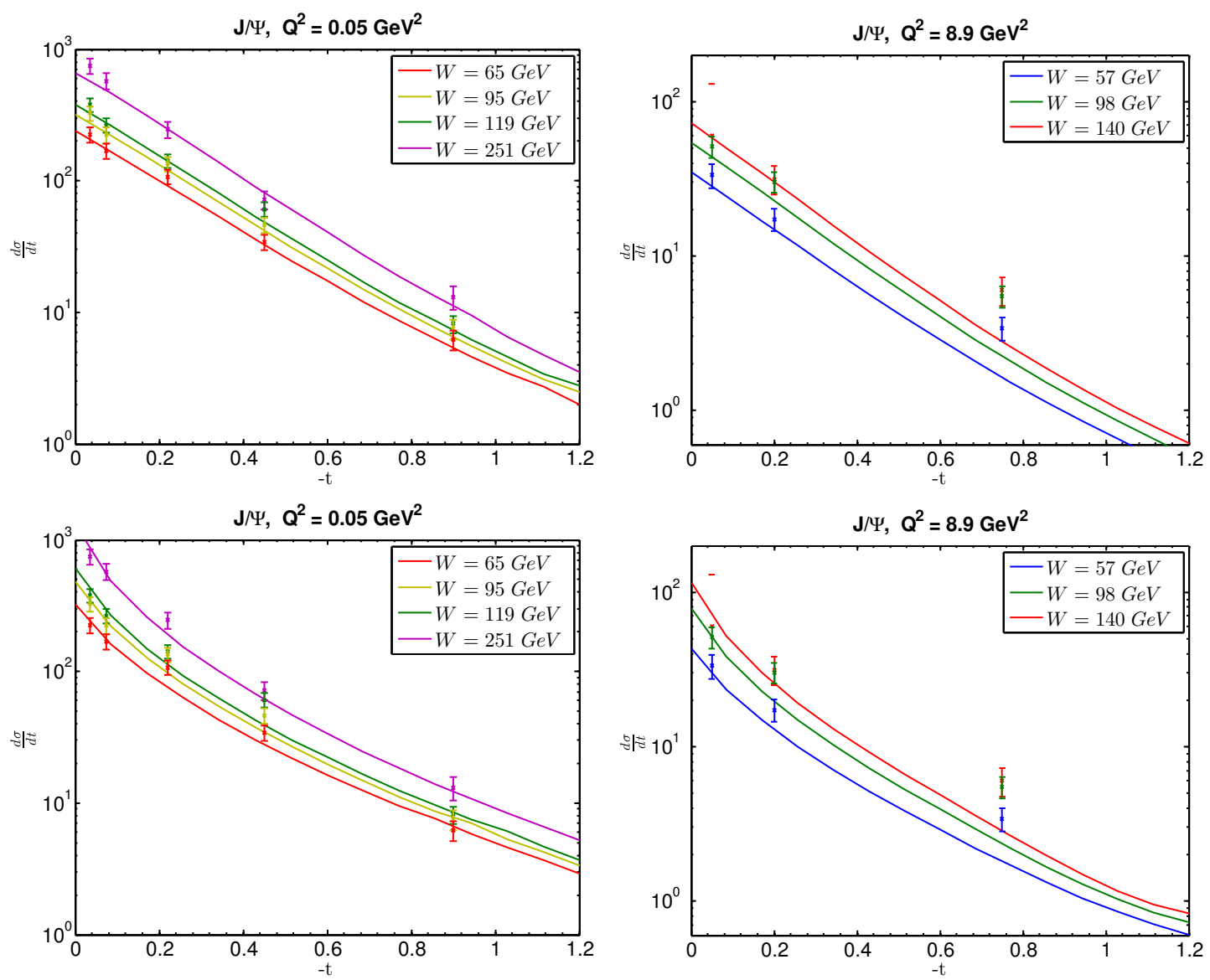

Figure 6. Differential cross section for the $J / \Psi$ meson for some of the $W$ and $Q^{2}$ values, using the hard-wall model (top) and the conformal model (bottom).

\section{Acknowledgments}

The authors are grateful to João Penedones and Chung-I Tan for helpful discussions. This work was partially funded by grants PTDC/FIS/099293/2008 and CERN/FP/116358/2010. Centro de Física do Porto is partially funded by FCT. The work of M.D. is supported by the FCT/Marie Curie Welcome II program. N.E. is grateful for the support of an STFC rolling grant. The research leading to these results has received funding from the People Programme (Marie Curie Actions) of the European Union's Seventh Framework Programme FP7/2007-2013/ under REA Grant Agreements No 269217 and No 317089.

\section{References}

[1] V.S. Fadin, E. Kuraev and L. Lipatov, On the Pomeranchuk Singularity in Asymptotically Free Theories, Phys. Lett. B 60 (1975) 50 [inSPIRE]. 
[2] E. Kuraev, L. Lipatov and V.S. Fadin, The Pomeranchuk Singularity in Nonabelian Gauge Theories, Sov. Phys. JETP 45 (1977) 199 [InSPIRE].

[3] I. Balitsky and L. Lipatov, The Pomeranchuk Singularity in Quantum Chromodynamics, Sov. J. Nucl. Phys. 28 (1978) 822 [inSPIRE].

[4] A. Martin, C. Nockles, M.G. Ryskin and T. Teubner, Small x gluon from exclusive J/psi production, Phys. Lett. B 662 (2008) 252 [arXiv:0709.4406] [INSPIRE].

[5] H.G. Dosch and E. Ferreira, Nonperturbative and perturbative aspects of photo- and electroproduction of vector mesons, Eur. Phys. J. C 51 (2007) 83 [hep-ph/0610311] [INSPIRE].

[6] C. Marquet, R.B. Peschanski and G. Soyez, Exclusive vector meson production at HERA from QCD with saturation, Phys. Rev. D 76 (2007) 034011 [hep-ph/0702171] [INSPIRE].

[7] A. Donnachie and P. Landshoff, Successful description of exclusive vector meson electroproduction, arXiv:0803.0686 [INSPIRE].

[8] S. Fazio, R. Fiore, L. Jenkovszky and A. Lavorini, Exclusive diffractive production of real photons and vector mesons in a factorized Regge-pole model with non-linear Pomeron trajectory, Phys. Rev. D 85 (2012) 054009 [arXiv:1109.6374] [INSPIRE].

[9] J. Berger and A.M. Stasto, Exclusive vector meson production and small-x evolution, JHEP 01 (2013) 001 [arXiv: 1205. 2037] [INSPIRE].

[10] A.H. Rezaeian, M. Siddikov, M. Van de Klundert and R. Venugopalan, Analysis of combined HERA data in the Impact-Parameter dependent Saturation model, Phys. Rev. D 87 (2013), no. 3034002 [arXiv:1212.2974] [INSPIRE].

[11] J. Forshaw and R. Sandapen, An AdS/QCD holographic wavefunction for the rho meson and diffractive rho meson electroproduction, Phys. Rev. Lett. 109 (2012) 081601 [arXiv: 1203.6088] [INSPIRE].

[12] R.C. Brower, J. Polchinski, M.J. Strassler and C.-I. Tan, The Pomeron and gauge/string duality, JHEP 12 (2007) 005 [hep-th/0603115] [INSPIRE].

[13] Y. Hatta, E. Iancu and A. Mueller, Deep inelastic scattering at strong coupling from gauge/string duality: The Saturation line, JHEP 01 (2008) 026 [arXiv:0710.2148] [INSPIRE].

[14] C. Ballon Bayona, H. Boschi-Filho and N.R. Braga, Deep inelastic scattering from gauge string duality in the soft wall model, JHEP 03 (2008) 064 [arXiv:0711.0221] [INSPIRE].

[15] C. Ballon Bayona, H. Boschi-Filho and N.R. Braga, Deep inelastic structure functions from supergravity at small $x$, JHEP 10 (2008) 088 [arXiv:0712.3530] [INSPIRE].

[16] C.B. Bayona, H. Boschi-Filho, N.R. Braga, M. Ihl and M.A. Torres, Generalized baryon form factors and proton structure functions in the Sakai-Sugimoto model, Nucl. Phys. B 866 (2013) 124 [arXiv:1112.1439] [INSPIRE].

[17] L. Cornalba and M.S. Costa, Saturation in Deep Inelastic Scattering from AdS/CFT, Phys. Rev. D 78 (2008) 096010 [arXiv:0804.1562] [INSPIRE].

[18] J.L. Albacete, Y.V. Kovchegov and A. Taliotis, DIS on a Large Nucleus in AdS/CFT, JHEP 07 (2008) 074 [arXiv: 0806.1484] [INSPIRE].

[19] E. Levin, J. Miller, B. Kopeliovich and I. Schmidt, Glauber-Gribov approach for DIS on nuclei in $N=4$ SYM, JHEP 02 (2009) 048 [arXiv:0811.3586] [INSPIRE]. 
[20] Y. Hatta, T. Ueda and B.-W. Xiao, Polarized DIS in $N=4$ SYM: Where is spin at strong coupling?, JHEP 08 (2009) 007 [arXiv: 0905.2493] [INSPIRE].

[21] Y.V. Kovchegov, Z. Lu and A.H. Rezaeian, Comparing AdS/CFT Calculations to HERA F(2) Data, Phys. Rev. D 80 (2009) 074023 [arXiv:0906.4197] [InSPIRE].

[22] E. Avsar, E. Iancu, L. McLerran and D. Triantafyllopoulos, Shockwaves and deep inelastic scattering within the gauge/gravity duality, JHEP 11 (2009) 105 [arXiv:0907.4604] [INSPIRE].

[23] L. Cornalba, M.S. Costa and J. Penedones, AdS black disk model for small-x DIS, Phys. Rev. Lett. 105 (2010) 072003 [arXiv: 1001.1157] [INSPIRE].

[24] E. Levin and I. Potashnikova, Inelastic processes in DIS and N=4 SYM, JHEP 08 (2010) 112 [arXiv: 1007.0306] [INSPIRE].

[25] R.C. Brower, M. Djuric, I. Sarcevic and C.-I. Tan, String-Gauge Dual Description of Deep Inelastic Scattering at Small-x, JHEP 11 (2010) 051 [arXiv:1007.2259] [INSPIRE].

[26] M. Betemps, V. Goncalves and J.T. de Santana Amaral, Diffractive deep inelastic scattering in an AdS/CFT inspired model: A phenomenological study, Phys. Rev. D 81 (2010) 094012 [arXiv: 1001.3548] [INSPIRE].

[27] R. Nishio and T. Watari, Investigating Generalized Parton Distribution in Gravity Dual, Phys. Lett. B 707 (2012) 362 [arXiv:1105.2907] [INSPIRE].

[28] R. Nishio and T. Watari, High-Energy Photon-Hadron Scattering in Holographic QCD, Phys. Rev. D 84 (2011) 075025 [arXiv:1105.2999] [INSPIRE].

[29] M.S. Costa and M. Djuric, Deeply Virtual Compton Scattering from Gauge/Gravity Duality, Phys. Rev. D 86 (2012) 016009 [arXiv:1201.1307] [INSPIRE].

[30] A. Karch and E. Katz, Adding flavor to AdS/CFT, JHEP 06 (2002) 043 [hep-th/0205236] [INSPIRE].

[31] M. Graña and J. Polchinski, Gauge/gravity duals with holomorphic dilaton, Phys. Rev. D 65 (2002) 126005 [hep-th/0106014] [INSPIRE].

[32] M. Bertolini, P. Di Vecchia, M. Frau, A. Lerda and R. Marotta, N=2 gauge theories on systems of fractional D3/D7 branes, Nucl. Phys. B 621 (2002) 157 [hep-th/0107057] [INSPIRE].

[33] M. Kruczenski, D. Mateos, R.C. Myers and D.J. Winters, Meson spectroscopy in AdS/CFT with flavor, JHEP 07 (2003) 049 [hep-th/0304032] [INSPIRE].

[34] J. Erdmenger, N. Evans, I. Kirsch and E. Threlfall, Mesons in Gauge/Gravity Duals - A Review, Eur. Phys. J. A 35 (2008) 81 [arXiv:0711.4467] [inSPIRE].

[35] T. Sakai and S. Sugimoto, Low energy hadron physics in holographic QCD, Prog. Theor. Phys. 113 (2005) 843 [hep-th/0412141] [INSPIRE].

[36] T. Sakai and S. Sugimoto, More on a holographic dual of QCD, Prog. Theor. Phys. 114 (2005) 1083 [hep-th/0507073] [INSPIRE].

[37] J. Erlich, E. Katz, D.T. Son and M.A. Stephanov, $Q C D$ and a holographic model of hadrons, Phys. Rev. Lett. 95 (2005) 261602 [hep-ph/0501128] [INSPIRE].

[38] L. Da Rold and A. Pomarol, Chiral symmetry breaking from five dimensional spaces, Nucl. Phys. B 721 (2005) 79 [hep-ph/0501218] [INSPIRE]. 
[39] S. Hong, S. Yoon and M.J. Strassler, On the couplings of vector mesons in AdS/QCD, JHEP 04 (2006) 003 [hep-th/0409118] [INSPIRE].

[40] L. Cornalba, M.S. Costa, J. Penedones and R. Schiappa, Eikonal Approximation in AdS/CFT: From Shock Waves to Four-Point Functions, JHEP 08 (2007) 019 [hep-th/0611122] [INSPIRE].

[41] L. Cornalba, M.S. Costa and J. Penedones, Eikonal approximation in AdS/CFT: Resumming the gravitational loop expansion, JHEP 09 (2007) 037 [arXiv:0707.0120] [INSPIRE].

[42] R.C. Brower, M.J. Strassler and C.-I. Tan, On the eikonal approximation in AdS space, JHEP 03 (2009) 050 [arXiv: 0707.2408] [inSPIRE].

[43] J. Bartels, J. Kotanski, A.-M. Mischler and V. Schomerus, Regge limit of R-current correlators in AdS Supergravity, Nucl. Phys. B 830 (2010) 153 [arXiv:0908.2301] [INSPIRE].

[44] L. Cornalba, M.S. Costa and J. Penedones, Deep Inelastic Scattering in Conformal QCD, JHEP 03 (2010) 133 [arXiv:0911.0043] [INSPIRE].

[45] R.C. Brower, M.J. Strassler and C.-I. Tan, On The Pomeron at Large 't Hooft Coupling, JHEP 03 (2009) 092 [arXiv:0710.4378] [INSPIRE].

[46] H1 collaboration, A. Aktas et al., Elastic J/ $\psi$ production at HERA, Eur. Phys. J. C 46 (2006) 585 [hep-ex/0510016] [inSPIRE].

[47] H1 collaboration, F. Aaron et al., Diffractive Electroproduction of rho and phi Mesons at HERA, JHEP 05 (2010) 032 [arXiv:0910.5831] [INSPIRE].

[48] ZEUS collaboration, S. Chekanov et al., Exclusive electroproduction of $\mathrm{J} /$ psi mesons at HERA, Nucl. Phys. B 695 (2004) 3 [hep-ex/0404008] [INSPIRE].

[49] ZEUS collaboration, J. Breitweg et al., Measurement of exclusive omega electroproduction at HERA, Phys. Lett. B 487 (2000) 273 [hep-ex/0006013] [INSPIRE].

[50] ZEUS collaboration, S. Chekanov et al., Exclusive electroproduction of phi mesons at HERA, Nucl. Phys. B 718 (2005) 3 [hep-ex/0504010] [INSPIRE].

[51] ZEUS collaboration, S. Chekanov et al., Exclusive rho0 production in deep inelastic scattering at HERA, PMC Phys. A 1 (2007) 6 [arXiv:0708.1478] [INSPIRE].

[52] H1 AND ZEUS collaboration, F. Aaron et al., Combined Measurement and QCD Analysis of the Inclusive $e^{ \pm} p$ Scattering Cross sections at HERA, JHEP 01 (2010) 109 [arXiv:0911.0884] [INSPIRE]. 